\title{
Experiencia en el manejo de litiasis renal y ureteral con ureteroscopio flexible y láser holmium en un hospital público de Bogotá: estudio retrospectivo
}

\section{Experience in the management of renal and ureteral calculi with holmium laser and flexible ureteroscope in a public hospital in Bogotá: A retrospective study}

\author{
Diana María Cerquera Cleves ${ }^{1}$ Andrés Felipe Puentes Bernal ${ }^{2}$

\begin{abstract}
Address for correspondence Diana María Cerquera Cleves, Residente 1.er año de urología, Universidad Nacional de Colombia-Hospital
\end{abstract} \\ Tunal, Bogotá, Colombia (e-mail: dianitamcc19@hotmail.com).
}

\footnotetext{
${ }^{1}$ Residente 1.er año de urología, Universidad Nacional de ColombiaHospital Tunal, Bogotá, Colombia

2 Urólogo, Universidad Nacional de Colombia, Servicio de urología, Hospital Tunal, Bogotá, Colombia
}

Urol Colomb 2018;27:74-80.

\section{Resumen}

Palabras-clave

- litiasis renal

- litiasis ureteral

- ureteroscopio flexible

- láser holmium

- radiografía de abdomen

- tomografía computarizada de abdomen simple

- tasa libre de cálculos

- complicaciones
Objetivos Discutir y evaluar la tasa libre de cálculos y las complicaciones de la ureterolitotomía endoscópica flexible con láser holmium en el manejo de cálculos ureterales y renales basada en la experiencia de un hospital público de III nivel en Bogotá.

Material and Métodos Estudio retrospectivo en una muestra poblacional en pacientes con litiasis renal y ureteral, tratados con ureteroscopio flexible y láser holmium, evaluándose la tasa libre de cálculos, complicaciones postoperatorias y otros parámetros. El seguimiento se realizó con radiografía de abdomen y urotac al mes del postoperatorio, la tasa libre de cálculos se definió como ausencia de cálculos residuales $<4 \mathrm{~mm}$ en la imagen control.

Resultados Se realizaron 44 procedimientos en 43 pacientes con edad promedio de 45 años, tiempo quirúrgico promedio 91,2 min, la tasa libre de cálculos fue del $84 \%$ (37/44) en un solo evento quirúrgico; solo un paciente requirió una segunda intervención con posterior tasa libre de cálculos del 100\%. La tasa libre de cálculos ureterales fue del $92 \%$ (24/26), cáliz superior 100\% (2/2), cáliz medio 100\%(1/1), cáliz inferior $78 \%(7 / 9)$ y calicial múltiple 50\% (3/6). El porcentaje de complicaciones fue del $6,8 \%$.

Conclusiones La ureterolitotomía endoscópica flexible con láser es un procedimiento efectivo y seguro en el manejo de litiasis renal y ureteral con baja incidencia de complicaciones. Observamos que en la mayoría de los pacientes con fragmentos residuales significativos estos se encontraban en cáliz inferior o se trataba de cálculos en múltiples cálices, explicándose por el difícil acceso al cáliz inferior y la carga litiásica. received

August 31, 2016

accepted

October 18, 2016

published online

January 10, 2018
DOI https://doi.org/

10.1016/j.uroco.2016.10.008.

ISSN 0120-789X.

eISSN 2027-0119.
Copyright (c) 2018, Sociedad Colombiana License terms de Urología. Publicado por Thieme Revinter Publicações Ltda., Rio de Janeiro, Brazil. Todos los derechos reservados.

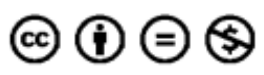


Abstract
Keywords
- renal stones
- ureteral stones
- flexible ureteroscopy
- holmium laser
- abdomen X-ray
- abdominal-pelvic computed axial tomography
- stone-free rate
- complications

Objective To discuss and review the stone free rate and complications of using flexible ureteroscopy and holmium laser lithotripsy for treating patients with kidney and ureteral stones, based on the experience in a level III public hospital in Bogotá. Materials and Methods A retrospective review was conducted on patients with renal and ureteral stones treated with flexible ureteroscopy and holmium laser, by analysing the stone-free rate and postoperative complications. X-rays or computed tomography was performed at 30 days post-operation to assess the effects of surgery, and the stonefree rate was defined as absence of residual stones $<4 \mathrm{~mm}$ on follow-up imaging.

Results A total of 44 procedures were performed on 43 patients with a mean age of 45 years. The mean operating time was 91.2 minutes, and the stone-free rate in a single surgical event was $84 \%$ (37/44). Only one patient required a second intervention, with a subsequent stone-free rate of $100 \%$. The ureteral stone-free rate was $92 \%(24 / 26)$, with upper calyx $100 \%(2 / 2)$, middle calyx $100 \%(1 / 1), 78 \%$ lower calyx (7/9), and multiple calyceal $50 \%(3 / 6)$. The complication rate was $6.8 \%$.

Conclusions Flexible ureteroscopy and holmium laser lithotripsy is an effective and safe procedure in the management of renal and ureteral stones, with a low incidence of postoperative complications. It was noted that most patients with significant residual fragments, they were in lower calyx or had multiple stones. This was explained by the difficult access to the lower calyx and stone burden.

\section{Introducción}

El uso de la ureteroscopia flexible se ha convertido en un método mínimamente invasivo, eficiente y seguro para la exploración de la vía urinaria superior; esta característica particular asociada al uso del láser como método de fragmentación permite el tratamiento de un sinnúmero de cálculos de diversa naturaleza mineralógica en cualquier localización con altas tasas libres de cálculos (TLC), constituyendo por lo tanto una buena alternativa a la nefrolitotomía percutánea y litotripsia extracorpórea. ${ }^{1}$

Desde su introducción por Marshall en 1964, se han venido publicando experiencias clínicas por parte de otros autores como Bagley et al., quienes fueron unos de los primeros en realizar un reporte de casos con 59 pacientes en quienes se usó el ureteroscopio flexible con fines diagnósticos, alcanzando la visualización del riñón hasta en el $88 \%$ de ellos y la inspección completa del sistema colector hasta en 23 pacientes de los reportados, mostrándolo como una posible alternativa para el diagnóstico de defectos de llenado intrarrenales. Sin embargo, hoy en día ha evolucionado de forma importante, llevando no solo a la posibilidad de su uso como herramienta diagnóstica, sino también de manejo para la extracción de cálculos ureterales e intrarrenales. $^{2}$

La prevalencia de cálculos renales ha aumentado en las últimas décadas especialmente en los últimos 20 años debido a tasas elevadas de obesidad, diabetes y síndrome metabólico, según reportes en EE. UU. Debido a esto, también se han venido implementando procedimientos quirúrgicos que promuevan el manejo de cálculos a nivel del tracto urinario superior, convirtiendo la ureterolitotomía endoscópica flexible con láser en una excelente opción para el manejo de cálculos de diversas composiciones y diferentes localizaciones. ${ }^{3}$
La TLC se considera como la ausencia de fragmentos litiásicos posterior al tratamiento o la presencia de fragmentos clínicamente insignificantes, entendiendo como «clínicamente insignificantes» aquellos fragmentos asintomáticos, no infecciosos, menores de $5 \mathrm{~mm}$ de diámetro persistentes después del tratamiento con litotripsia extracorpórea, y ha sido adoptada para referirse a la medición del éxito posterior a otro tipo de procedimientos mínimamente invasivos para el manejo de cálculos renales., ${ }^{3,4}$ Sin embargo hoy en día se cuestiona esta definición debido a que hasta el $40 \%$ de los pacientes con cálculos residuales de $5 \mathrm{~mm}$ pueden cursar con un evento clínico sintomático que puede requerir reintervención. Por este motivo Khurshid et al. realizaron una revisión de los reportes de casos de los pacientes manejados con ureteroscopio flexible para el tratamiento de cálculos renales considerando que los fragmentos $<$ de $4 \mathrm{~mm}$ son los que presentan menor posibilidad de reintervención por presentar sintomatología. ${ }^{3}$

En contraposición a investigaciones previas que demostraban TLC similares en el manejo de cálculos mayores $>20 \mathrm{~mm}$ por medio de litotripsia extracorpórea o ureteroscopio flexible con láser, estudios posteriores que incluyeron un número mayor de pacientes demostraron que el uso de ureteroscopio flexible logra mayor TLC que la litotripsia extracorpórea. Sin embargo, la nefrolitotomía percutánea y los procedimientos percutáneos mínimamente invasivos siguen presentando las mayores TLC, especialmente en cargas litiásicas altas ( $>20 \mathrm{~mm}$ ), teniendo en cuenta que esto es a expensas de la prolongación en el tiempo de hospitalización, aumento en el sangrado y complicaciones en general. $^{5-8}$

La nefrolitotomía percutánea es usada especialmente en el manejo de cálculos $>20 \mathrm{~mm}$, sin embargo la tasa de fragmentos residuales puede llegar hasta el $83 \%$; debido a esto se han evaluado los diferentes métodos mínimamente 
invasivos para el manejo de la reintervención de la litiasis residual, demostrando que tanto el ureteroscopio flexible como la litotricia extracorpórea pueden ser utilizados para este fin, no obstante, el manejo con equipo flexible y láser tiene mayor TLC que la litotripsia extracorpórea, eligiéndolo como método de elección por algunos estudios. ${ }^{9}$

Anteriormente los estudios no demostraban resultados significativos cuando comparaban el manejo por medio de ureteroscopio flexible y litotripsia extracorpórea en los cálculos renales $<10 \mathrm{~mm}$ ubicados en el cáliz inferior; sin embargo, hoy en día se postula que puede ser considerado como la primera línea de manejo para este tipo de cálculos y adicionalmente para cálculos de hasta $20 \mathrm{~mm}$ en esta localización, presentando una TLC mayor y con el requerimiento de menos intervenciones en comparación con la litotripsia extracorpórea, sin existir mayor riesgo de complicaciones. ${ }^{10-14}$

El uso de la camisa ureteral en ureteroscopia flexible ha sido muy discutido pues presenta riesgos y beneficios; por un lado, muestra grandes beneficios disminuyendo el trauma ureteral por el paso repetitivo del equipo flexible por el uréter, mejora el flujo de irrigación facilitando la visualización y disminuyendo el tiempo operatorio, aumenta la durabilidad del equipo flexible y finalmente disminuye la presión en la pelvis renal llevando a una reducción en el riesgo de infección y lesión renal. Sin embargo, en contraposición se ha demostrado que dependiendo del calibre de la camisa puede causar isquemia de la pared ureteral y lesión del uréter, haciendo necesario el uso rutinario de catéter postoperatorio. ${ }^{15-18}$

Existen diversas investigaciones que reportan los resultados de diferentes centros con respecto al uso de ureteroscopio flexible con láser holmium para el manejo de cálculos ureterales y renales. En Colombia las muestras más grandes fueron evidenciadas en diferentes estudios entre los que se encuentra un estudio retrospectivo en el 2009, con evidencia de TLC del $86 \%$ y con fragmentos residuales significativos en pacientes que presentaban litiasis múltiple o cálculos $>$ de $10 \mathrm{~mm}$, y complicaciones postoperatorias en el 9\% de los pacientes. Posteriormente en el 2014 se llevó a cabo un segundo estudio retrospectivo con TLC del 92,8\% de las cuales la tasa más baja fue en los cálculos mayores de $10 \mathrm{~mm}$ (85\%), sin embargo obtuvieron muy buenos resultados en los procedimientos en cálculos múltiples (TLC: 95\%), sin complicaciones reportadas. ${ }^{19-21}$

Considerando las ventajas descritas del procedimiento y el hecho de que cada vez se está extendiendo más su uso en varios centros en Colombia, se decidió realizar este estudio en un hospital público de Bogotá cuyo objetivo fue evaluar de forma retrospectiva los procedimientos de de litiasis renal y ureteral proximal manejados con ureteroscopio flexible y láser holmium, con el fin de describir los resultados obtenidos y compararlos con los reportados en la literatura, así como evaluar si en esta población existen variables demográficas, clínicas o quirúrgicas que se relacionen con la proporción de TLC.

\section{Metodología}

Se realizó un estudio retrospectivo con datos desde diciembre de 2012 hasta enero de 2016, en un hospital público de Bogotá, en el cual se incluyeron pacientes mayores de 18 años con diagnóstico de urolitiasis en localización calicial, ureteral proximal o calicial y ureteral múltiple con densidades $>1.000 \mathrm{UH}$, manejados con ureteroscopio flexible y láser holmium. Los procedimientos fueron realizados por 2 urólogos diferentes en cada cirugía. El diagnóstico de la litiasis urinaria fue realizado por medio de tomografía axial computarizada simple de abdomen (urotac), se utilizaron ureteroscopios flexibles marca Wolf de $9 \mathrm{Fr}$ y posteriormente Storz de 7,5 Fr con fibra láser holmium de 200 y 272 um. Se definió como TLC a la ausencia de cálculos o fragmentos residuales $<4 \mathrm{~mm}$ posteriores al manejo en pacientes asintomáticos. Se utilizó la clasificación de Dindo-Clavien del 2004 para evaluar las complicaciones postoperatorias. ${ }^{22}$ El seguimiento se realizó a los 15 y a los 30 días por medio de una radiografía de abdomen simple para los cálculos radioopacos o urotac, evaluándose la TLC y las complicaciones postoperatorias.

Otras variables estudiadas en estos pacientes fueron el tiempo quirúrgico, el uso o no de camisa ureteral durante el procedimiento u otras herramientas endoscópicas, el tiempo de hospitalización y el promedio de edad, sexo y lateralidad. Se compararon las variables demográficas, clínicas y quirúrgicas para valorar si existían diferencias significativas respecto a la TLCy al uso o no de camisa de acceso ureteral. Se emplearon las pruebas de $U$ de Mann Whitney para evaluar diferencias en variables cuantitativas y la exacta de Fisher para variables cualitativas. Se consideraron significativos valores de $\mathrm{p}<0,05$.

\section{Resultados}

En el periodo de 3 años se llevaron a cabo 106 procedimientos en 101 pacientes, de los cuales se excluyeron 58 pacientes porque no cumplieron con los criterios de inclusión; 4 de ellos correspondían a procedimientos láser por calcificación de catéter JJ y estrechez ureteral y 54 no tenían historia clínica completa, o no se logró adecuado seguimiento postoperatorio. Finalmente, al estudio ingresaron 43 pacientes que cumplieron con los criterios de inclusión, quienes fueron sometidos a 44 procedimientos, debido a la necesidad de reintervención de uno ellos por fragmentos residuales significativos.

Se realizaron 21 procedimientos en mujeres y 23 en hombres, con una edad promedio de 44 años; de estos, 31 procedimientos fueron del lado derecho y 13 del izquierdo, no se realizaron procedimientos bilaterales. El tiempo quirúrgico en promedio fue de $91 \mathrm{~min}$ (desviación estándar 28,8), siendo de 180 min la cirugía de mayor duración en un paciente con un cálculo de $40 \mathrm{~mm}$ en cáliz inferior, en quien se logró fragmentación completa. La localización más frecuente correspondió al uréter proximal (59\%), seguido de los cálculos caliciales únicos inferiores $(20 \%)$ y posteriormente de los cálculos múltiples en varias localizaciones (14\%). Las características demográficas, clínicas e intraoperatorias de toda la población se describen en la - Tabla 1.

Se trataron cálculos $\geq 20 \mathrm{~mm}$ en el $27 \%$ de los pacientes. La mediana de la carga litiásica para los cálculos únicos de localización calicial inferior fue de $13 \mathrm{~mm}$, calicial medio de $15 \mathrm{~mm}$ y superior de $13 \mathrm{~mm}$; y para los ureterales proximales 
Tabla 1 Características de los pacientes

\begin{tabular}{|c|c|}
\hline & $n=44$ \\
\hline Edad; media (DE) & $44,2(13,1)$ \\
\hline \multicolumn{2}{|l|}{ Sexo; $n(\%)$} \\
\hline Hombres & $23(52)$ \\
\hline Mujeres & $21(48)$ \\
\hline Unilaterales; $n$ (\%) & $44(100)$ \\
\hline Derecho & $31(70)$ \\
\hline Izquierdo & $13(30)$ \\
\hline \multicolumn{2}{|l|}{ Localización; n (\%) } \\
\hline Superior & $2(4,5)$ \\
\hline Medio & $1(2,5)$ \\
\hline Inferior & $9(20)$ \\
\hline Uréter proximal & $26(59)$ \\
\hline Múltiple & $6(14)$ \\
\hline Carga litiásica; media (DE) & $16,1(8,6)$ \\
\hline Carga litiásica $\geq 20$ mm; n (\%) & $12(27)$ \\
\hline$T L C<4 \mathrm{~mm} ; n(\%)$ & $37(84)$ \\
\hline Tiempo operatorio; media (DE) & $91(28,8)$ \\
\hline Complicaciones; n (\%) & $3(6,8)$ \\
\hline Uso de camisa ureteral; $n$ (\%) & $20(45)$ \\
\hline Uso de catéter JJ; n (\%) & $40(91)$ \\
\hline Stone Cone; n (\%) & $8(18)$ \\
\hline Procedimiento ambulatorio; $n$ (\%) & $32(73)$ \\
\hline
\end{tabular}

Abreviaciones: DE, desviación estándar; TLC, tasa libre de cálculos.

fue de $12 \mathrm{~mm}$ en su diámetro transverso. En la litiasis múltiple se encontró una mediana de carga litiásica de 29 mm (23-35). La TLC total fue del 84\%, en el uréter proximal fue del $92 \%$ ( 24 / 26) y a nivel renal fue del $100 \%$ en cálices superiores $(2 / 2)$ y medio (1/1) y del $78 \%$ en cáliz inferior (7/9), para cálculos caliciales y ureterales múltiples el porcentaje fue del 50\% (3/6), que incluía a 2 pacientes con cargas litiásicas altas (35 y 40 $\mathrm{mm}$ ) y un tercer paciente en el que no se logró la extracción completa de los cálculos en uréter proximal por la evidencia de estrechez ureteral distal y media secundaria; este último paciente requirió posteriormente extracción abierta de los cálculos y reconstrucción ureteral con flap de Boari por la magnitud de las múltiples estrecheces. En la -Tabla 2 se relacionan la localización de los cálculos con la carga litiásica, la TLC y las complicaciones postoperatorias.

Se presentaron 3 complicaciones que representaron el 6,8\% de la muestra. La primera fue Clavien I por un paciente con litiasis múltiple que posterior al procedimiento cursó con cólico renal, requiriendo de analgesia intravenosa, con expulsión espontánea de los fragmentos residuales; la segunda fue Clavien II por infección de vías urinarias complicada en el paciente con estrechez ureteral mencionado previamente; y la tercera fue Clavien IIla consistente en un absceso perirrenal que requirió drenaje percutáneo.

La camisa ureteral de 11/13 Fr fue usada en 20 (45\%) procedimientos sin evidenciar diferencias significativas ni en la TLC, ni en cuanto a tiempo quirúrgico o complicaciones, cuando se comparó con los pacientes en quienes no se utilizó camisa ureteral ( - Tabla 3). Se utilizó dispositivo antirretropulsión (Stone Cone) en el 18\% de los pacientes con litiasis ureteral y adicionalmente se colocó catéter JJ a más del $90 \%$ de los pacientes (40/44) por el edema ureteral que se presenta secundario al procedimiento; este fue retirado en los controles. El uso de la canastilla permitió el reposicionamiento de cálculos especialmente de cáliz inferior en algunos procedimientos, logrando así mejor manipulación del cálculo con el equipo y por consiguiente mayor facilidad para la fragmentación completa. Ante el hallazgo de cálculos en el interior de un divertículo renal en un paciente, se usó primero el azul de metileno para realizar la tinción del divertículo y posteriormente se realizó la fragmentación y extracción completa con el equipo, sin complicaciones posteriores.

La presencia de cálculos múltiples y una carga litiásica mayor fueron características comunes en los pacientes que no lograron alcanzar la TLC, además el tiempo operatorio también fue mayor en este tipo de pacientes. En el resto de las variables no se identificaron diferencias estadísticamente significativas (- Tabla 4).

En este estudio se evaluó la utilización del ureteroscopio flexible para el manejo de cálculos con localización renal, ureteral proximal y con carga litiásica múltiple, evidenciando TLC favorables, similares a las reportadas en la literatura, especialmente en los cálculos con ubicación en uréter proximal $(92 \%)$ cáliz superior y medio $(100 \%){ }^{19}$ No obstante, la fragmentación de los cálculos múltiples demostraron TLC menores a las reportadas; esto puede

Tabla 2 Relación entre localización de cálculos, carga litiásica, TLC y complicaciones postoperatorias

\begin{tabular}{|c|c|c|c|c|c|}
\hline Localización & $\mathrm{n}$ & $\begin{array}{l}\text { Carga litiásica; } \\
\text { mediana (RIC) }\end{array}$ & Carga litiásica > 20 mm; n (\%) & TLC n (\%) & Complicaciones n (\%) \\
\hline Superior & 2 & $13(13-13)$ & $0(0)$ & $2(100)$ & - \\
\hline Medio & 1 & 15 & $0(0)$ & $1(100)$ & - \\
\hline Inferior & 9 & $13(10-24)$ & $3(33)$ & $7(78)$ & $1(11)$ \\
\hline Uréter proximal & 26 & $12(10-14)$ & $3(12)$ & $24(92)$ & - \\
\hline Múltiple & 6 & $29(23-35)$ & $6(100)$ & $3(50)$ & $2(33)$ \\
\hline
\end{tabular}

Abreviaciones: RIC, rango intercuartílico; TLC, tasa libre de cálculos. 
Tabla 3 Tabla comparativa entre pacientes en los que se utilizó camisa de acceso ureteral durante el procedimiento vs. pacientes en los que no se usó

\begin{tabular}{|l|l|l|l|}
\hline & $\begin{array}{l}\text { Pacientes con camisa } \\
\text { de acceso ureteral } \mathbf{n}=\mathbf{2 0}\end{array}$ & $\begin{array}{l}\text { Pacientes sin camisa } \\
\text { de acceso ureteral } \mathbf{n}=\mathbf{2 4}\end{array}$ & $\mathbf{P}^{*}$ \\
\hline Complicaciones; $\mathbf{n}(\%)$ & $2(10)$ & $1(4)$ & 0,583 \\
\hline Tiempo quirúrgico (min); mediana (RIC) & $90(60-120)$ & $90(70-120)$ & 0,990 \\
\hline TLC; $\mathbf{n}(\%)$ & $16(80)$ & $21(87,5)$ & 0,683 \\
\hline
\end{tabular}

Abreviaciones: RIC: rango intercuartílico; TLC: tasa libre de cálculos.

*Valores de $\mathrm{p}$ correspondientes a la prueba $\mathrm{U}$ de Mann-Whitney para comparaciones cuantitativas y prueba exacta de Fisher para las cualitativas.

deberse a que presentaban una carga litiásica mayor, y a la baja muestra de pacientes en este subgrupo adicionando sus características específicas.

En los pacientes evaluados, la TLC en carga litiásica $>20 \mathrm{~mm}$ fue del $60 \%$ en comparación con la litiasis $<20 \mathrm{~mm}$ que obtuvo desenlaces favorables (92\%), demostrando que en esta muestra la carga o el volumen litiásico alto fue un factor predisponente para presentar TLC bajas. Estos mismos resultados también han sido demostrados previamente en estudios retrospectivos que evaluaban los factores que influían en la predicción de la TLC. Adicionalmente se ha demostrado que el tratamiento de los cálculos $>20 \mathrm{~mm}$ manejados con ureteroscopio flexible con láser presentan menor TLC que los manejados con nefrolitotomía percutánea, sin embargo la presencia de complicaciones y de días de hospitalización es menor con el uso de procedimientos mínimamente invasivos. ${ }^{23}$

Con respecto a los cálculos localizados en cáliz inferior, pueden presentar riesgo de generar cólico renal y por este motivo en lo posible se deben clasificar los pacientes para evaluar cuáles se beneficiarían del manejo expectante; sin embargo en nuestro estudio no se dio manejo expectante llevando a todos los pacientes a fragmentación con ureteroscopio flexible con láser, demostrando TLC del 78\% en litiasis en esta posición que se explica por el difícil acceso a los cálices inferiores por medio del equipo flexible, y la dificultad para su expulsión espontánea habitual. Estos resultados se

Tabla 4 Diferencias entre los pacientes libres de cálculo (TLC) vs. pacientes con persistencia de fragmentos residuales significativos

\begin{tabular}{|c|c|c|c|}
\hline & $\begin{array}{l}\mathrm{TLC}(<4 \mathrm{~mm}) \\
\mathrm{n}=37\end{array}$ & $\begin{array}{l}\text { Fragmentos residuales } \\
\text { significativos ( }>4 \mathrm{~mm} \text { ) } \\
\mathrm{n}=7\end{array}$ & $\mathrm{p}$ \\
\hline \multicolumn{4}{|l|}{ Localización; $n$ (\%) } \\
\hline Superior & $2(5)$ & $0(0)$ & \\
\hline Medio & $1(3)$ & $0(0)$ & \\
\hline Inferior & $7(19)$ & $2(28,5)$ & \\
\hline Uréter proximal & $24(65)$ & $2(28,5)$ & \\
\hline Múltiple & $3(8)$ & $3(43)$ & $<0,05^{*}$ \\
\hline \multicolumn{4}{|l|}{ Carga litiásica } \\
\hline Carga litiásica; mediana (RIC) & $13(10-15)$ & $25(12-40)$ & $<0,05^{*}$ \\
\hline Carga litiásica > 20 mm; n (\%) & $7(19)$ & $5(71)$ & $<0,05^{*}$ \\
\hline Carga litiásica $<20$ mm; n (\%) & $30(81)$ & $2(29)$ & \\
\hline Tiempo operatorio; mediana (RIC) & $90(60-100)$ & $120(105-120)$ & $<0,05^{*}$ \\
\hline Complicaciones; n (\%) & $1(3)$ & $2(28,5)$ & \\
\hline Uso de camisa ureteral; $n$ (\%) & $16(43)$ & $4(57)$ & \\
\hline Uso de catéter JJ; n (\%) & $34(92)$ & $6(86)$ & \\
\hline Stone Cone; n (\%) & $8(22)$ & $0(0)$ & \\
\hline Procedimiento ambulatorio; $n$ (\%) & $27(73)$ & $5(71)$ & \\
\hline
\end{tabular}

Abreviaciones: RIC: rango intercuartílico; TLC: tasa libre de cálculos.

*Valores de p estadísticamente significativos según la prueba $\mathrm{U}$ de Mann-Whitney para comparaciones cuantitativas y prueba exacta de Fisher para las cualitativas 
relacionan con los conseguidos por estudios retrospectivos los cuales hablan de una alta incidencia de cálculos en el cáliz inferior debido a diferentes factores pielocaliciales que disminuían las TLC; ellos reportan que uno de los factores más importantes además del volumen litiásico es el ángulo infundibulopélvico $<30^{\circ}$, que tiene una gran influencia en los resultados posterior al procedimiento. ${ }^{24-27}$

El riesgo de complicaciones postoperatoria presentadas en el estudio (6,8\%) no sobrepasa al de la literatura en general, demostrando que además de ser un procedimiento con buenos resultados, es un manejo seguro. ${ }^{28}$

El tiempo operatorio en el estudio fue en promedio de 91 min similar al reportado en otras series, sin embargo, se evidenció un aumento promedio del tiempo especialmente en pacientes que presentaban cálculos $>20 \mathrm{~mm}$ a diferencia de los que mostraban cálculos $<20 \mathrm{~mm}$, probablemente secundario a la alta carga litiásica en el primer grupo.

En nuestro estudio se usó camisa ureteral durante el procedimiento en 20 pacientes (45,5\%), no obstante, no demostró diferencias significativas con respecto a la TLC, complicaciones o tiempo quirúrgico que fueron resultados muy similares a los reportados en una muestra colombiana y en otros estudios en la literatura. ${ }^{29}$

Durante el procedimiento se usó dispositivo antirretropulsión en el $18 \%$ de los pacientes y se realizó colocación de catéter JJ postoperatorio aproximadamente al 90\% de ellos; aunque no se presentaron estrecheces ureterales, y solo se reportó un cólico renal en el seguimiento, no hubo una relación significativa en nuestra muestra en relación con el aumento de la TLC. ${ }^{30}$

\section{Conclusions}

En nuestro estudio, el uso de ureteroscopio flexible y láser en el tratamiento de la litiasis renal y ureteral única con diámetros promedio de hasta $20 \mathrm{~mm}$ logró TLC equiparables con las reportadas en la literatura con un porcentaje bajo de complicaciones. Los cálculos múltiples y aquellos ubicados en el cáliz inferior continúan siendo un reto terapéutico con TLC menores a las reportadas para otras localizaciones.

\section{Responsabilidades Éticas}

\section{Protección de personas y animales}

Los autores declaran que para esta investigación no se han realizado experimentos en seres humanos ni en animales.

\section{Confidencialidad de los dadtos}

Los autores declaran que han seguido los protocolos de su centro de trabajo sobre la publicación de datos de pacientes.

\section{Derecho a la privacidad y consentimiento informado}

Los autores declaran que en este artículo no aparecen datos de pacientes.

Conflicto de intereses

No existe conflicto de intereses.

\section{Bibliografía}

1 Chen FS, Xu FB, Liu FN, et al. Improved effectiveness and safety of flexible ureteroscopy for renal calculi $(<2 \mathrm{~cm})$ : A retrospective study. Can Urol Assoc J 2015;9:E273-E277

2 Giusti FG, Proietti FS, Villa FL, et al. Current standard technique for modern flexible ureteroscopy: Tips and tricks. Eur Urol 2016; 70:188-194

3 Ghani FK, Wolf FJ, Wolf FJ. What is the stone-free rate following flexible ureteroscopy for kidney stones? Nat Rev Urol 2015; $12: 281-288$

4 Lingeman FJ, Newman FD, Mertz FJ, et al. Extracorporeal shock wave lithotripsy: The Methodist Hospital of Indiana experience. J Urol 1986;135:1134-1137

5 Hyams FE, Munver FR, Bird FV, Uberoi FJ, Shah FO. Flexible ureterorenoscopy and holmium laser lithotripsy for the management of renal stone burdens that measure 2 to $3 \mathrm{~cm}$ : A multi-institutional experience. J Endourol 2010;24:1583-1588

6 Aboumarzouk FO, Monga FM, Kata FS, Traxer FO, Somani FB. Flexible ureteroscopy and laser lithotripsy for stones $>2 \mathrm{~cm}$ : A systematic review and meta-analysis. J Endourol 2012;26:1257-1263

7 De FS, Autorino FR, Kim FF, et al. Percutaneous nephrolithotomy versus retrograde intrarenal surgery: A systematic review and meta-analysis. Eur Urol 2015;67:125-137

8 Takazawa FR, Kitayama FS, Tsujii FT. Successful outcome of flexible ureteroscopy with holmium laser lithotripsy for renal stones $2 \mathrm{~cm}$ or greater. Int J Urol 2012;19:264-267

9 Xu FG, Wen FJ, Li FZ, et al. A comparative study to analyze the efficacy and safety of flexible ureteroscopy combined with holmium laser lithotripsy for residual calculi after percutaneous nephrolithotripsy. Int J Clin Exp Med 2015;8:4501-4507

10 Sener FN, Bas FO, Sener FE, et al. Asymptomatic lower pole small renal stones: shock wave lithotripsy, flexible ureteroscopy, or observation? A prospective randomized trial. Urology 2015;85:33-37

11 El-Nahas FA, Ibrahim FH, Youssef FR, Sheir FK. Flexible ureterorenoscopy versus extracorporeal shock wave lithotripsy for treatment of lower pole stones of $10-20 \mathrm{~mm}$. BJU Int 2012; 110:898-902

12 Burr FJ, Ishii FH, Simmonds FN, Somani FB. Is flexible ureterorenoscopy and laser lithotripsy the new gold standard for lower pole renal stones when compared to shock wave lithotripsy: Comparative outcomes from a University hospital over similar time period. Cent European J Urol 2015;68:183-186

13 Kumar FA, Vasudeva FP, Nanda FB, Kumar FN, Das FM, Jha FS. A prospective randomized comparison between shock wave lithotripsy and flexible ureterorenoscopy for lower caliceal stones $\leq 2 \mathrm{~cm}$ : A single-center experience. J Endourol. 2015; 29:575-579

14 Pearle FM, Lingeman FJ, Leveillee FR, et al. Prospective randomized trial comparing shock wave lithotripsy and ureteroscopy for lower pole caliceal calculi $1 \mathrm{~cm}$ or less. J Urol 2008;179(I 5 Suppl):S69-S73

15 Kaplan FA, Lipkin FM, Scales FC, Preminger FG. Use of ureteral access sheaths in ureteroscopy. Nat Rev Urol 2016;13:135-140

16 Auge FB, Pietrow FP, Lallas FC, Raj FG, Santa-Cruz FR, Preminger FG. Ureteral access sheath provides protection against elevated renal pressures during routine flexible ureteroscopic stone manipulation. J Endourol 2004;18:33-36

17 Stern FJ, Yiee FJ, Park FS. Safety and efficacy of ureteral access sheaths. J Endourol 2007;21:119-123

18 Berquet FG, Prunel FP, Verhoest FG, Mathieu FR, Bensalah FK. The use of a ureteral access sheath does not improve stone-free rate after ureteroscopy for upper urinary tract stones. World J Urol 2014;32:229-232

19 Redondo FC, Ramón de Fata FF, Gimbernat FH, Meilán FE, Andrés FG, Angulo FJ. Retrograde intrarenal surgery with holmium-YAG laser lithotripsy in the primary treatment of renal lithiasis. Actas Urol Esp 2015;39:320-326 
20 Gallo FJ, Uribe FC. Ureterorrenoscopia flexible con Ho:YAG láser en el manejo de la litiasis del tracto urinario superior. Descripción de los primeros 80 casos. Urol Colom 2009;3:83-92

21 Shek Padilla FA, Otero Andrade FF, Agressot Guerra FW, et al. Nefrolitotomía retrógrada flexible láser de holmium: primeros 100 casos. Urol Colomb. 2014;23:103-108

22 Dindo FD, Demartines FN, Clavien FP. Classification of surgical complications: A new proposal with evaluation in a cohort of 6336 patients and results of a survey. Ann Surg 2004;240:205-213

23 Hyams FE, Monga FM, Pearle FM, et al. A prospective, multiinstitutional study of flexible ureteroscopy for proximal ureteral stones smaller than $2 \mathrm{~cm}$. J Urol 2015;193:165-169

24 Donaldson FJ, Lardas FM, Scrimgeour FD, et al. Systematic review and meta-analysis of the clinical effectiveness of shock wave lithotripsy, retrograde intrarenal surgery, and percutaneous nephrolithotomy for lower-pole renal stones. Eur Urol 2015; 67:612-616

25 Ito FH, Sakamaki FK, Kawahara FT, et al. Development and internal validation of a nomogram for predicting stone-free status after flexible ureteroscopy for renal stones. BJU Int 2015;115:446-451

26 Ito FH, Kawahara FT, Terao FH, et al. Evaluation of preoperative measurement of stone surface area as a predictor of stone-free status after combined ureteroscopy with holmium laser lithotripsy: A single-center experience. J Endourol 2013;27:715-721

27 Inoue FT, Murota FT, Okada FS, et al. Influence of pelvicaliceal anatomy on stone clearance after flexible ureteroscopy and holmium laser lithotripsy for large renal stones. J Endourol 2015;29:998-1005

28 Akman FT, Binbay FM, Ozgor FF, et al. Comparison of percutaneous nephrolithotomy and retrograde flexible nephrolithotripsy for the management of 2-4 cm stones: A matched-pair analysis. BJU Int 2012;109:1384-1389

29 Neira FI, Celis FE, Zapata FM. Seguridad de la ureteroscopia flexible sin camisa de acceso ureteral. Urol Colomb 2014;23:171-176

30 Eisner FB, Dretler FS. Use of the Stone Cone for prevention of calculus retropulsion during holmium: YAG laser lithotripsy: Case series and review of the literature. 2009;82:356-366 